\title{
Desafios da educação permanente no acolhimento em saúde
}

\author{
Challenges of continuing education in health care \\ Retos de la educación sanitaria permanente \\ Mariane Pereira de Gouvea ${ }^{1 *}$, Claúdia Vieira Carnevalle ${ }^{1,2}$, Roudom Ferreira Moura ${ }^{1,2,3}$.
}

\section{RESUMO}

Objetivo: Identificar as contribuições da educação permanente, a partir do acolhimento qualificado, para a melhoria da qualidade do atendimento aos usuários do Sistema Único de Saúde. Revisão Bibliográfica: A partir da análise de 20 artigos, ficou evidenciado que a educação permanente para profissionais de saúde é de suma importância, seja para os profissionais atuantes na produção de serviços como para os gestores e profissionais de apoio, tendo em vista que a educação no trabalho é considerada como instrumento estratégico para transformações efetivas para a melhoria da qualidade dos serviços de saúde. O Programa Nacional de Educação Permanente em Saúde visa promover saúde com base em preceitos do acolhimento. Considerações finais: Entende-se que educar de maneira que traga ao profissional de saúde a capacidade de reflexão crítica, baseada na realidade do cenário de saúde brasileiro, é o que se espera da educação permanente. Porém, a educação permanente está em segundo plano em ambiente desfavorável no cenário de saúde.

Palavras-chave: Educação permanente, Acolhimento, Humanização da assistência.

\section{ABSTRACT}

Objective: To identify the contributions of permanent education, based on qualified reception, to improve the quality of care provided to users of the Brazilian Unified Health System. Bibliographic Review: From the analysis of 20 articles, it was evidenced that permanent education for health professionals is of paramount importance, both for professionals working in the production of services and for managers and support professionals, considering that education at work is considered as a strategic instrument for effective transformations to improve the quality of health services. The National Program for Permanent Education in Health aims to promote health based on the precepts of welcoming. Final considerations: It is understood that educating in a way that brings health professionals the capacity for critical reflection, based on the reality of the Brazilian health scenario, is what is expected from permanent education. However, permanent education is in the background in the health scenario.

Keywords: Permanent education, Reception, Humanization of assistance.

\section{RESUMEN}

Objetivo: Identificar las contribuciones de la educación permanente, basada en una recepción calificada, para mejorar la calidad de la atención brindada a los usuarios del Sistema Único de Saúde. Revisión bibliográfica: Del análisis de 20 artículos, se evidenció que la educación permanente para profesionales de la salud es de suma importancia, tanto para profesionales que trabajan en la producción de servicios como para gerentes y profesionales de apoyo, considerando que la educación en el trabajo es considerado como una herramienta estratégica para transformaciones efectivas para mejorar la calidad de los servicios de salud. El Programa Nacional de Educação Permanente em Saúde tiene como objetivo promover la salud sobre la base de los preceptos de anfitrión. Consideraciones finales: Se entenderá que educar de una manera que brinde al profesional de la salud la capacidad de reflexión crítica, basada en la realidad del escenario de salud brasileño, es lo que se espera de la educación permanente. Sin embargo, la educación permanente está en según plan en el escenario de la salud.

Palabras clave: Educación permanente, Recepción, Humanización de la asistencia.

\footnotetext{
1 Universidade São Judas Tadeu (USJT), São Paulo - SP. * E-mail: marianegouveaa@gmail.com

2 Secretaria de Estado da Saúde de São Paulo, São Paulo - SP.

${ }^{3}$ Faculdade de Saúde Pública da Universidade de São Paulo (FSP), São Paulo - SP.
} 


\section{INTRODUÇÃO}

O Sistema Único de Saúde (SUS) é norteado por princípios básicos, dentre eles, a visão integral do usuário em todos os aspectos como ser humano e não apenas como um portador de uma patologia a ser tratada. Nesse sentido, pressupõe-se ao profissional de saúde que seja capacitado de forma adequada e, dessa forma, esteja apto a olhar, analisar e compreender o usuário em toda sua complexidade como ser humano, em sua total integralidade (ARAÚJO D, et al., 2007).

Com a atuação profissional, alinhada à falta de atenção, a subjetividade do sujeito é possível entrelaçar a outros problemas que persistem no SUS como a desvalorização profissional, a precarização das relações de trabalho, o baixo investimento em processos de educação permanente em saúde desses trabalhadores, a pouca participação na gestão dos serviços e o frágil vínculo com os usuários. Assim, em um cenário complexo no que diz respeito a qualidade do atendimento sentida pelos profissionais e usuários, se faz necessário transformações tanto no modelo de atenção como no de gestão. Nesta perspectiva, o Ministério da Saúde desenvolve, a fim de fortalecer o SUS como política pública de saúde, a Política Nacional de Humanização da Atenção e Gestão no SUS (HumanizaSUS) (BRASIL, 2016a).

A humanização deve ser vista não como um programa, mas como política pública que atravessa e transversaliza as diferentes ações e instâncias gestoras do SUS traduzindo os princípios do mesmo e os adequando para a operacionalização com práticas orientadas pela experiência do trabalhador e usuário igualando essas experiências e sua importância, trazendo o protagonismo e autonomia dos sujeitos e coletivos. Humanizar significa valorizar a participação dos diferentes sujeitos no processo de saúde, trabalhadores, usuários e gestores (BRASIL, 2016a).

No Brasil, foi necessário a criação de uma política para alinhar a prática profissional aos ideais do SUS. O acolhimento é uma diretriz da política nacional que se transversaliza com as demais e se potencializa como forma de inclusão para desconstruir o modelo biomédico centrado na patologia, priorizando a escuta ativa e o cuidado para a percepção do sujeito e de todas suas demandas e necessidades (BRASIL, 2016b).

Para Carotta et al. (2009), a educação permanente em saúde, utilizada como ferramenta pelos gestores de saúde, possibilita a reflexão crítica relativas às práticas de atenção, gestão e formação. $O$ uso dessa ferramenta auxilia na obtenção das mudanças nas relações, processos, no sistema de saúde, nos usuários e profissionais, por se tratar de um processo educativo envolvendo o trabalho.

Frente as premissas apresentadas no tangente ao SUS - doutrina da integralidade, HumanizaSUS política de humanização para as práticas de assistência à saúde pública, a fim de obter a melhor forma de acolhimento junto aos usuários, através da educação permanente de profissionais que trabalham na saúde, surgem diversos desafios para o sistema universal de saúde do Brasil que justificam o presente estudo (LIMA LD, et al., 2018). Diante do exposto, este trabalho teve como objetivo identificar as contribuições da educação permanente, a partir do acolhimento qualificado, para a melhoria da qualidade do atendimento aos usuários do SUS.

\section{REVISÃO BIBLIOGRÁFICA}

\section{Política Nacional de Educação Permanente (PNEPS)}

O Ministério da Saúde do Brasil (MS) realizou a implantação do Política Nacional de Educação Permanente em Saúde (PNEPS) em 2004 e, a partir desse momento, três esferas de governo passaram a ser responsáveis pela estimulação e desenvolvimento de ações de cunho educativo entre os profissionais e usuários dentro das instituições de saúde, ou, nas comunidades. Dessa forma, o MS buscou melhorar o processo de trabalho e o relacionamento com os usuários, melhorando, em consequência disso, o atendimento (SOUZA GG, et al., 2017).

Devido ao surgimento de novas epidemias e das dificuldades frente às mesmas, falta de investimentos e recurso, demandas nos setores de trabalho, de planejamento de atividades intersetoriais e interdisciplinares, a educação permanente em saúde, infelizmente, tem seguido em segundo plano na maioria das instituições, sendo assim, ela acaba sendo uma atividade focalizada sem resultados produtivos. Nessas instituições, a 
educação em saúde não é trabalhada como uma ferramenta complementar para auxiliar na criação de novos atores sociais, capazes de resolver a problemática que se apresenta, hoje, nos serviços de saúde (SOUZA GG, et al., 2017).

Outro fator importante para o atual quadro da educação permanente em saúde nas instituições é a burocracia da operacionalização que a gestão disponibiliza, o que tem contribuído para desmotivar muitos profissionais. Muitos deles deixam de acreditar nos resultados da PNEPS que tem como missão promover a saúde baseando-se no diálogo, informação e educação para uma reflexão crítica e aprendizagem significativa (CARDOSO IM, 2012).

Segundo Sousa MST, et al. (2015), os profissionais de saúde não compreendem os conceitos de educação permanente, acreditando em processos de atualizações e não em estratégia de participação ativa de propor, reordenar e transformar as práticas. É necessário que os profissionais, gestores e facilitadores vivenciem e reconheçam a PNEPS, suas diretrizes e bases metodológicas para estratégias assertivas que favoreçam um cenário de mudança de atitude.

\section{Educação permanente em saúde no Brasil}

Discussões envolvendo a América Latina e Brasil em meados da década de 70, a respeito da educação permanente na saúde, norteadas a partir da conceituação de educação envolvendo o desenvolvimento de uma percepção crítica e raciocínio reflexivo para o crescimento do homem como cidadão, recomendada pela Organização Pan-Americana de Saúde (OPAS), destacou os métodos de trabalho no enaltecimento das experiências vividas e ações de saúde dos atores sociais.

Tal discussão foi a base para a formulação de uma proposta que visa reorientar os processos de educação em saúde, visando a aprendizagem no processo de trabalho. Os debates serviram, ainda, para desencadear significativas mudanças nos conceitos de educação profissional vigentes até hoje (CAMPOS KFC, et al., 2017).

No Brasil, no mesmo momento em que a discussão sobre a educação permanente ganha espaço, influenciado pela OPAS, desenvolvendo-se cada vez mais, surge também à discussão sobre a reforma sanitária, onde as Conferências Nacionais de Saúde (CNS) introduziram, de forma respectiva, Conferências Nacionais de Recursos Humanos de 1986 e 1993, voltado para a área da saúde, trazendo indícios da necessidade de formação orientada à realidade da saúde. As CNS defendiam que os profissionais de saúde têm papel fundamental nas mudanças que são necessárias na atenção à saúde dos cidadãos (CAMPOS KFC, et al., 2017).

Em 1988, com a criação do SUS, houve a oportunidade de expansão dos serviços de atenção primária à saúde, serviço dinamicamente inserido na vida da comunidade, demandado a necessidade de novas abordagens e ação dos profissionais. Ficaram evidentes, também, a pressão e demandas da população que de maneira tradicional, os profissionais de saúde ainda não estavam aptos a responder como esperado. Com isso, a insatisfação, cobrança e insuficiência no modo agir em serviços de saúde propiciaram um clima cultural e político que possibilitava a expansão de diversas iniciativas e propostas de melhoria no ensino (VASCONCELOS EM, et al., 2016).

Dessa forma, Almeida JRS, et al. (2016) complementam a ideia de que é necessária a realização de aperfeiçoamento dos profissionais de saúde adequada a realidade da população. "Ressalta-se que a reorientação da formação profissional em saúde deve ser um movimento de fortalecimento do SUS, portanto, a formação em serviço passa a ter papel fundamental na metodologia pedagógica".

Estudos afirmam que não apenas os profissionais de saúde já atuantes devem participar ativamente da PNEPS para o SUS, como, também, docentes, discentes, e gestores de ensino e informação científicotecnológica, a fim de produzir uma política setorial e de interface (FRANCO HL e CARNEIRO AT, 2017).

De acordo com, Justo AM, et al. $(2018,2019)$ que destacam a importância da educação permanente em saúde orientados por meio da Portaria de Consolidação no 2 de 28 de setembro de 2017, onde a educação permanente se integra ao SUS em seu cotidiano de maneira prática, exercendo influência direta nas instituições formadoras dos profissionais de saúde. 
As diretrizes incorporadas pela PNEPS propiciam campos para a formação de profissionais qualificados e que sejam capazes de considerar os processos críticos e reflexivos a respeito do trabalho, não se tornando apenas reprodutores de processos estabelecidos. Assim, o perfil do profissional que se almeja precisa conjugar capacidade reflexiva e problematizadora ao conhecimento teórico/acadêmico específico de sua prática de atuação.

\section{Acolhimento e Humanização}

Acolher é um termo que define a forma, o como e de que maneira se receber, ou, é recebido, abrigo garantido gratuitamente e hospitalidade, exercício de ouvir com atenção o outro, dar crédito (acreditar), admitir, aceitar. Acolher é possibilitar o acesso universal dos serviços de saúde a todos os usuários que necessitam (SOUZA ABS, et al., 2016).

O acolhimento deve estar contido em todas as relações de cuidado que envolver trabalhadores de saúde e usuários, nas ações de receber e escutar os indivíduos. Essas ações podem ocorrer em diferentes lugares (recepção, sala de espera, consultório, residências, entre outros), sendo parte das atribuições que são comuns a todos os membros da equipe multiprofissional, o que favorece a ampliação do acesso ao serviço (CASARIN N, et al., 2017).

Dessa forma, conforme já se exigia os princípios básicos do SUS, como a universalidade, equidade e participação social, foi criado em 1994, o Programa Saúde da Família (PSF), com o objetivo claro de contribuir com a reorientação do modelo assistencial partindo da atenção básica, fazendo com que a mesma adote uma dinâmica diferente na sua atuação, definindo responsabilidades entre os serviços de saúde e a população.

Assim, o PSF propõe-se como uma prestação da assistência à saúde que envolvia uma equipe multiprofissional de forma que trabalhassem de maneira integral, contínua, que trazia resultados e que fosse de boa qualidade, considerando sempre os fatores de vulnerabilidade e riscos nos quais a população estava inserida a determinado território. O programa, também, teve a finalidade da criação do vínculo profissional com os usuários de saúde, responsabilizando, assim, aos mesmos, a responsabilidade de estimular a população a adotar boas práticas de saúde por meio da educação (OLIVEIRA CVS, et al., 2016).

Ainda segundo Oliveira CVS, et al. (2016), em 2007 foi realizada uma análise com os primeiros 12 municípios participantes do PSF e foi constatado problemas decorrentes ao acesso as unidades de saúde. Isso se dava por conta da dificuldade de integração entre Unidades de Saúde da Família (USF) e as equipes da atenção básica e, também, com a falta de integração da atenção primária e com a atenção secundária (média complexidade) e terciária (alta complexidade) o que impedia a continuidade da atenção prestada.

Atualmente, o PSF é definido como Estratégia de saúde da Família (ESF), ao invés de programa, visto que se trata de uma estratégia de reorganização da atenção primaria e não prevê tempo de finalização. Com a ESF sobrecarregada, era difícil a integração com outros setores para a articulação de estratégias de promoção de saúde.

Assim, o MS, identificando os problemas enfrentados sentiu a necessidade de inserir a humanização como uma das Políticas de Governo, onde todo o SUS passaria por mudanças e todas as ações, de todas as instancias estariam vinculadas a práticas humanizadas de acordo com as diretrizes da pela Portaria $n^{\circ}$ 2.488/2011 que instituiu a Política Nacional de Humanização (PNH), também, conhecida como HumanizaSUS (BRASIL, 2011).

Motta BFB, et al. (2014) descrevem que nessa proposta a ideia é "qualificar as práticas de gestão e atenção em saúde", onde o acolhimento é a diretriz de maior relevância do ponto de vista ético - por ser pautado por meio da subjetividade do usuário, político - por ser dotado de compromissos coletivos, onde todos são envolvidos no processo de produção em saúde, e estéticos - por propor a dignidade à vida e ao viver de cada indivíduo.

Assim, o acolhimento não se trata de ações isoladas e pontuais, mas sim um processo responsável onde se busca a criação de um vínculo que se forma a partir da escuta dos problemas do usuário, das trocas de informações entre usuário e profissionais, mútuos conhecimentos dos direitos e deveres e decisões que são tomadas de acordo com a necessidade de cada um, acertada de comum acordo entre as partes (BRASIL, 2010). 
Affonso PHB e Bernardo MH (2015) concluem que uma das novas propostas do acolhimento, foi a ampliação da clínica, que pode ser realizada por outros profissionais que não seja médico, incluindo diferentes abordagens a diversos problemas de saúde. Nesse cenário, as propostas de acolhimento estariam de acordo com o contexto político da época, onde a proposta não é mudar as condições que fazem as pessoas adoecerem, uma vez que isso não é possível.

Dessa forma, a ética que deveria ser empregada na prestação dos serviços de saúde torna-se apenas objeto de propaganda sem sentido nenhum, ou, até negativo quando se fala da promoção de saúde. A ideia de implantar o acolhimento no sistema de saúde do Brasil foi inovadora, sendo que serve de apoio para as necessidades dos pacientes, pois os profissionais podem aplicar diferentes possibilidades de cuidado (AFFONSO PHB e BERNARDO MH, 2015).

Não obstante Coutinho LRP, et al. (2015) pontua que desde o princípio, diversos problemas foram evidenciados para a adoção das práticas do acolhimento humanizado. O número insuficiente de profissionais representa um dos reflexos negativos para os usuários que não possuem um atendimento que reflita suas necessidades e tenha efeito. Esse reflexo negativo também atinge os profissionais que ficam cansados e incapacitados de atender a todos com exatamente os mesmos critérios de qualidade, o que causa a insatisfação tanto desses profissionais como dos usuários.

\section{Desafios da Educação Permanente e Humanização}

Vista como uma nova forma de transformar os serviços, a educação permanente em saúde, procura atuar com os trabalhadores em saúde, dando-lhes subsídios para que eles possam solucionar problemas da comunidade por meio da formulação de estratégias. A proposta é aperfeiçoar o método educacional em saúde, onde as atividades profissionais são o objeto a serem transformado, garantindo que os princípios básicos do SUS como a equidade, universalidade e integralidade sejam colocadas em prática efetiva, melhorando assim por consequência a qualidade do atendimento à população (VEPO AA, et al., 2015).

O termo educação permanente surgiu na França em 1955, mas teve registro oficial em 1956 em um documento do Ministro Educacional a respeito do prolongamento da escolaridade obrigatória e a reforma do ensino público no país. Só no fim da década de 1960 o termo passou a ser explorado pela Organização das Nações Unidas para a Educação, Ciência e Cultura (UNESCO), onde fundamentava-se dentro da teoria do capital humano, que defende a ideia de que o ser humano qualificado é um capital mais importante em uma cadeia produtiva econômica e desenvolvida do país (LEMOS CLS, 2016).

A denominação de educação permanente em saúde surgiu na metade da década de 1980 , e foi disseminada por meio do Programa de Desenvolvimento de Recursos Humanos da OPAS. Nessa época, a OPAS sugere a diferenciação entre os termos educação permanente e educação continuada, sendo a última considerada mais reducionista, porém, sem ser unanime em meio acadêmico (LEMOS CLS, 2016).

Independentemente do termo utilizado, Coswosk ED, et al. (2017) afirmam que a educação é uma ação humana de suma importância, necessária à existência do indivíduo como ser humano e que garante o funcionamento de todas as atividades de produção. Dessa forma, os autores atentam para a importância da formação dos atores sociais, da qual o processo de educação depende e permite que eles estejam aptos a atuar em meio à sociedade com autonomia suficiente para não repetir ações por pura automação. Porém, isso é mais complicado na prática.

A Educação Permanente, em sua implementação, apresenta algumas dificuldades no que diz respeito ao cenário de saúde pública no Brasil, começando pela separação existente entre a execução da PNEPS e sua formulação, o que demonstra claramente a distorção da realidade que os formuladores da política possuem em relação a gestão pública de saúde (CAMPOS KFC, et al., 2017).

Um dos desafios da falta de vivência da realidade é achado principalmente nas instancias do SUS, onde os profissionais não conseguem cumprir nem a missão básica de levar assistência às comunidades e nem suportam a sobrecarga que o ensino lhe acrescenta. A dificuldade está na redução do pessoal de apoio que dificulta a prática da educação no trabalho como instrumento gerencial no processo de trabalho. Além disso, o desafio da liberação de recursos, também, é um dos desafios a serem enfrentados, já que a falta de recursos 
implica em profissionais mal remunerados e inviabiliza a implantação de ações de promoção em saúde por parte dos gestores. Dessa forma, é inviável estimular o desenvolvimento de profissionais a promover saúde para os usuários do SUS (CAMPOS KFC, et al., 2017).

O desafio da educação permanente em saúde está na estimulação do desenvolvimento dos profissionais no que diz respeito a sua responsabilidade na participação desse processo. Sendo assim, é necessário reavaliar métodos educativos na prevenção e promoção dos processos sistematizados e participativos, usando de cenário o espaço de produção, em situações que fomentam o pensar e agir dentro dos processos de trabalho (SILVA LAA, et al., 2017).

Tavares MFL, et al. (2016) demonstram que a promoção da saúde tem enfrentado problemas no que diz respeito à criação e a realização das políticas públicas saudáveis, a formação de ambientes favoráveis, 0 fortalecimento da ação comunitária, o amadurecimento de habilidades pessoais e a reorientação dos serviços.

Lima LPS e Ribeiro MRR (2016) concluíram de que a Educação Permanente passou a ser, apenas, um discurso de ideologias, diferentemente do que a UNESCO pregava, que almejava implantar educação para a vida toda, assim compromisso que foi assumido por vários países, inclusive o Brasil.

De acordo com os autores, a educação permanente para os trabalhadores dos serviços de saúde serve apenas para os tornarem mais rentáveis e adaptáveis a diversidade que as mudanças econômicas e industriais causam. Porém, a política de saúde trouxe consigo a proposta da educação permanente que tem o foco de atender as necessidades da sociedade, mediante as constantes mudanças no mundo, procurando aprimorar indivíduo em suas potencialidades e ainda visando melhorar a qualidade do atendimento.

Para Roman C, et al. (2017) é de fundamental importância que o ensino em saúde incorpore os avanços das metodologias pedagógicas no processo de ensino-aprendizagem, onde a base da mudança estaria na criação de novas diretrizes para a que teoria e prática se integrem, rompendo o modelo tradicional de ensino na formação clínica e básica.

Dessa forma, a universidade tem o dever de implementar em suas atividades, metodologias que propiciem o desenvolvimento do senso crítico, da habilidade de reflexão e da participação efetiva dos estudantes na busca pela construção do conhecimento, para que os mesmos se tornem profissionais comprometidos, trabalhando de forma integrada com a equipe multiprofissional.

No demais, esse estudo tem como fator limitante de pesquisa ter encontrado menor número de artigos relacionando as duas temáticas propostas, educação permanente e acolhimento em saúde no período dos últimos 10 anos. Na literatura foi encontrado em maior número trabalhos que não relacionam esses dois temas em seu estudo.

\section{CONSIDERAÇÕES FINAIS}

A educação permanente em saúde está se transformando ao longo dos anos e ganhando, cada vez mais, espaço com a criação dos programas governamentais que preconizam a humanização. São evidentes as dificuldades, ainda, enfrentadas pela educação permanente, entre a principal delas está o baixo investimento e de sua falta efetiva. Diante do exposto, o acolhimento ganha espaço, visto que sua importância está no fato de o mesmo ser a forma pela qual os profissionais de saúde e a sociedade entram em contato para assim, firmarem a prestação de um serviço. A atenção quanto subjetividade do sujeito no encontro de saúde fica a critério do profissional, assim devem existir ações motivadoras para o empoderamento profissional e a monitorização do processo de acolhimento com a garantia de condições de trabalho e desenvolvimento adequadas para a efetividade no processo de saúde. Sugere-se mais trabalhos que relacionam os dois temas aplicados nesse estudo: educação permanente e acolhimento em saúde, haja a vista a lacuna apresentada nesse estudo. Em suma, acredita-se que quanto mais capacitado for o indivíduo, mais sucesso, em qualquer processo de transformação aplicado, terá.

\section{AGRADECIMENTOS E FINANCIAMENTO}

Agradeço a Diretoria de Pesquisa e Pós-Graduação Stricto Sensu da Universidade São Judas Tadeu responsável por essa Iniciação Cientifica, meus orientadores e familiares pelo apoio. 


\section{REFERÊNCIAS}

1. AFFONSO PHB, BERNARDO MH. A vivência de profissionais do acolhimento em unidades básicas de saúde: uma acolhida desamparada. Trabalho, Educação e Saúde. 2015; 13(1): 23-43.

2. ALMEIDA JRS, et al. Educação Permanente em Saúde: uma estratégia para refletir sobre o processo de trabalho. Revista da ABENO. 2016; 16(2): 7-15.

3. ARAÚJO D, et al. Formação de profissionais da saúde na perspectiva da integralidade. Revista Baiana de Saúde Pública. 2007; 31(1): 20-31.

4. BRASIL. Ministério da Saúde. Documento Base para Gestores e Trabalhadores do SUS. Ministério da Saúde, Secretaria de Atenção a Saúde, Núcleo Técnico da Política Nacional de Humanização - 4.ed., 6. Reimpr.- Brasília: Editora do Ministério da Saúde, 2016a.

5. BRASIL. Ministério da Saúde. Acolhimento na GESTÃO E O TRABALHO EM SAÚDE. Ministério da Saúde, Secretaria de Atenção à Saúde, Núcleo Técnico da Política Nacional de Humanização - 1.ed.- Brasília: Editora do Ministério da Saúde, 2016b.

6. BRASIL. Ministério da Saúde. Cadernos HumanizaSUS. Brasília, DF: Ministério da Saúde. 2010.

7. BRASIL. Portaria ${ }^{\circ} 2.488$ de 21 de outubro de 2011. Aprova a Política Nacional de Atenção Básica, estabelecendo a revisão de diretrizes e normas para a organização da Atenção Básica, para Estratégia Saúde da Família (ESF) e o Programa de Agentes comunitários de Saúde (PACS). Diário Oficial da União. 2011 out.24; seção 1.

8. CAMPOS KFC, et al. Educação permanente nos serviços de saúde. Esc Anna Nery. Minas Gerais, 2017; 21(4).

9. CAMPOS KFC, et al. Educação Permanente: avanços, desafios para a gestão em saúde no Brasil. Investigação Qualitativa em Educação. Minas Gerais, 2016; 1.

10. CARDOSO IM. "Rodas de educação permanente" na atenção básica de saúde: analisando contribuições. Saude soc. São Paulo, 2012; 21(1).

11. CAROTTA F, et al. Educação Permanente em Saúde: uma estratégia de gestão para pensar, refletir e construir práticas educativas e processos de trabalho. Saúde Soc. São Paulo, 2009; 18(1).

12. CASARIN N, et al. Fórum de Humanização: potente espaço para educação permanente de trabalhadores da Atenção Básica. Rev. Saúde Debate. Rio de Janeiro, 2017; 41(114): 718-728.

13. COUTINHO AS, et al. O acolhimento na Atenção Básica em saúde: relações de reciprocidade entre trabalhadores e usuários. Rev. Saúde Debate. Rio de Janeiro, 2015; 39(104).

14. COUTINHO LRP, et al. Acolhimento na Atenção Primária à Saúde: revisão integrativa. Revista Saúde em Debate. Rio de Janeiro, 2015; 39(105): 514-524.

15. COSWOSK ED, et al. Educação continuada para o profissional de saúde no gerenciamento de resíduos de Saúde. Revista Brasileira de Análises Clínicas - RBAC. UNEB. Bahia, 2017.

16. FRANCO HL, CARNEIRO AT. A evolução da educação permanente em saúde e sua influência no atendimento humanizado ao paciente. Revista Internacional de Debates da Administração Pública. São Paulo, $2017 ; 2(1): 1$-11.

17. JUSTO AM, et al. Bases para diálogos e reflexões em Educação Permanente em Saúde. Superintendência de Educação em Saúde - Rio de Janeiro, 2018/2019.

18. LEMOS CLS. Educação Permanente em Saúde no Brasil: educação ou gerenciamento permanente? Ciência \& Saúde Coletiva. 2016; 21(3): 913-922.

19. LIMA LD, et al. Sistema Único de Saúde: 30 anos de avanços e desafios. Cadernos de Saúde Pública. 2018; 34(7).

20. 20.LIMA LPS, RIBEIRO MRR. A competência para Educação Permanente em Saúde: percepções de coordenadores de graduações da saúde. Revista de Saúde Coletiva. 2016; 26(2).

21. MOTTA BFB, et al. O acolhimento em Saúde no Brasil: uma revisão sistemática de literatura sobre o tema. Rev. SBPH. Rio de Janeiro, 2014;17(1).

22. OLIVEIRA CVS, et al. Desafios do acolhimento na Estratégia Saúde da Família: Uma revisão integrativa. Saúde em Redes. Pernambuco, 2016; 2(2): $211-225$.

23. ROMAN C, et al. Metodologias ativas de ensino-aprendizagem no processo de ensino em saúde no Brasil: uma revisão narrativa. Clin Biomed Res. 2017; p. 349-357.

24. SILVA LAA, et al. Educação permanente em saúde na atenção básica: percepção dos gestores municipais de saúde. Revista Gaúcha de Enfermagem. 2017.

25. SOUZA ABS, et al. Acolhimento realizado na atenção básica pela equipe de enfermagem ao paciente portador de transtorno mental: uma revisão teórica. Revista Amazônia Science \& Health. Gurupi.TO, 2016.

26. SOUZA GG, et al. A importância da Educação Permanente em Saúde no fortalecimento das representações sociais. Universidade Federal do Vale do São Francisco. Programa de Pós-graduação em Ciências da Saúde e Biológicas PPGCSB. II CONBRACIS - Congresso Brasileiro de Ciências da Saúde. 2017.

27. SOUSA MST, et al. A percepção dos enfermeiros sobre educação permanente em saúde no contexto da estratégia saúde da família de Sobral (CE). Revista Interfaces: Saúde, Humanas e Tecnologia. 2015; 3(1).

28. TAVARES MFL, et al. A promoção da saúde no ensino profissional: desafios na Saúde e a necessidade de alcançar outros setores. Escola Nacional de Saúde Pública Sergio Arouca, Fiocruz. Rio de Janeiro, 2016.

29. VASCONCELOS EM, et al. A contribuição da Educação Popular para a formação profissional em saúde. Interface. São Paulo, 2016; 835-8.

30. VEPO AA, et al. Educação permanente em saúde: a chave para a qualificação na atenção integra. Anais do VII Salão Internacional de Ensino, Pesquisa e Extensão - Universidade Federal do Pampa. Uruguaiana - RS, 2015. 